\title{
Addendum to: An Iterated Logarithm Law for Families of Brownian Paths
}

Z. Wahrscheinlichkeitstheor. Verw. Geb. 70, 341-344 (1985)

\author{
Raoul LePage ${ }^{1}$ and Bertram M. Schreiber ${ }^{2} \star^{2}$ \\ ${ }^{1}$ Department of Statistics and Probability, Michigan State University, East Lansing, \\ MI 48824, USA \\ ${ }^{2}$ Department of Mathematics, Wayne State University, Detroit, MI 48202, USA
}

After the papers [2] and [3] had gone to press, the authors generalized these results to Brownian paths in separable Banach spaces and strengthened the limiting process to almost sure convergence. Through a communication with A. de Acosta we have learned that the latter result follows by an easy modification of the techniques used to prove Theorem 9 of [1], which gives priority for this result to the authors of [1].

Our proof (with V. Goodman) of the Banach space result is of some interest. The outer law, together with the one-dimensional inner law for the line passing through an arbitrary point on the surface of the unit ball of the kernel space, are used to deduce the full inner law by a simple compactness and scaling argument.

\section{References}

1. Acosta, A. de, Kuelbs, J.: Limit theorems for moving averages of independent random vectors. Z. Wahrscheinlichkeitstheor. Verw. Geb. 64, 67-123 (1983)

2. LePage, R., Schreiber, B.M.: An iterated logarithm law for families of Brownian paths. Z. Wahrscheinlichkeitstheor. Verw. Geb. 70, 341-344 (1985)

3. LePage, R., Schreiber, B.M.: A square root law for diffusing particles. In: Probability on Banach Spaces V (Proc. Conf., Medford, MA, 1984). Lect. Notes Math. 1153. Berlin Heidelberg New York: Springer 232-238 (1985)

* Supported in part by NSF Grant MCS 82-00786 